\title{
Maximum Packing Channel Assignment in Cellular Networks
}

\author{
Ashutosh Kulshreshtha and Kumar N. Sivarajan, Member, IEEE
}

\begin{abstract}
We study the performance of the maximum packing channel assignment algorithm (MPA) in channelized cellular networks. MPA is a greedy algorithm, which rejects a call only when it is forced to do so, even if this involves rearrangement of channels assigned to the ongoing calls, without dropping any of them. We ignore handoffs and model the channel reuse constraints in the cellular network by a hypergraph [1]. As the traffic and the number of channels are scaled together, we get a limiting regime where the blocking probability in the cells can be computed by solving a nonlinear optimization problem. The carried traffic in this limiting case is an upper bound on the performance of MPA for practical finite-channel systems. We show that the performance of MPA in a finite-channel cellular system can be closely approximated by considering a simple fixed-routing circuit-switched network. Thus, the finite-channel performance of MPA can be studied using methods well known in the area of circuit-switched networks. We compare the performance of MPA with other asymptotically optimal algorithms and demonstrate its optimality for low and moderate offered traffic. We envisage MPA as a practical channel assignment algorithm, for moderate size systems, and suggest approximations to reduce its complexity.
\end{abstract}

Index Terms - Channel assignment algorithm, channel reassignment, hypergraph, linear program, maximal independent set, maximum packing algorithm, optimization problem.

\section{INTRODUCTION}

W E STUDY the performance of channel assignment algorithms in cellular networks. We will see that in a system in which the load in comparison to the capacity is low or moderate, a greedy channel assignment algorithm performs better than several other algorithms. Thus, traffic control techniques such as call-admission control may be unnecessary in well-engineered cellular systems. However, under heavy load, the performance of the same algorithm is quite poor.

In a cellular telephone system, the territory is divided into mutually disjoint cells (usually depicted as being hexagonal). Each cell is served by a base station at its center. The total system bandwidth is divided into channels, which are characterized by a frequency slot or a time slot or a combination of both. Our analysis applies only to channelized cellular

Manuscript received September 18, 1997; revised August 3, 1998. This work was carried out at the Indian Institute of Science and supported in part by the Department of Electronics, Government of India, through its Education and Research Network (ERNET) Project.

A. Kulshreshtha is with the IBM Solutions Research Center, Indian Institute of Technology, Delhi 110 016, India (e-mail: kashutos@in.ibm.com).

K. N. Sivarajan is with the Electrical Communication Engineering Department, Indian Institute of Science, Bangalore 560 012, India (e-mail: kumar@ece.iisc.ernet.in).

Publisher Item Identifier S 0018-9545(99)04036-0. telephone systems and is not applicable, for example, to codedivision multiple-access (CDMA) systems. Each mobile user needs a channel to communicate with the base station in its geographical vicinity. The same channel can be simultaneously used in different cells, subject to certain restrictions, for example, cochannel interference should be less than a particular limit. This is termed channel reuse and the restrictions are called reuse constraints.

When a call requests service in a given cell, either a channel which does not violate the channel reuse constraints, is assigned to the call, or else the call is blocked. One objective of channel assignment policy is to minimize the number of calls denied service. Recent research has proposed and analyzed many channel assignment schemes, which can be broadly classified into three major categories.

\section{A. Fixed Channel Assignment Algorithm (FCAA)}

The set of channels is partitioned into subsets and these subsets are permanently assigned to cells for their exclusive use, without violating reuse constraints.

\section{B. Dynamic Channel Assignment Algorithm (DCAA)}

Any channel can be used in any cell, where it is needed. When a new call arrives, it is assigned a channel which does not violate the channel reuse constraints. If such a channel is not available, the call is blocked. Channels assigned to calls in progress are not disturbed. DCAA overcomes the inability of FCAA to cope with temporary stochastic variations in traffic.

\section{Maximum Packing Algorithm (MPA)}

MPA is a greedy channel assignment algorithm, which accepts a call whenever possible, even if, this involves rearrangement of the channels assigned to calls already in progress. But no call in progress is dropped to accommodate a new call. MPA was first introduced by Everitt et al. in [2]. Note that there can be other dynamic channel assignment algorithms that permit reassignments but are different from MPA.

Example 1: To distinguish MPA from DCAA, consider the linear three-cell system shown in Fig. 1, with two channels available, labeled 1 and 2 . Assume that channel reuse constraints do not allow channels to be used simultaneously in neighboring cells but allow them to be used in cells 1 and 3 simultaneously. Assume that at some point in time, channel 1 has been assigned to a call in cell 1 and channel 2 to a call in cell 3. In this case, DCAA will block a call request in cell 2, as none of the channels can be assigned to it. But MPA will 


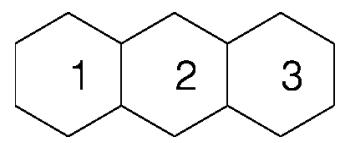

Fig. 1. The linear three-cell system.

pack the two ongoing calls into one channel and will assign the other channel to the call in cell 2.

DCAA and MPA have natural appeal due to better performance in low and moderate traffic and the ability to automatically cope with changing traffic patterns. We may speculate that MPA is an optimal algorithm, and it gives a tight bound on system performance achievable by any DCA algorithm. Though this is true in some cellular systems, e.g., symmetrical cellular systems with equal load, in general it may be true only up to a particular load. Most of the studies on DCAA and MPA are based on simulations; see [3], [4], and references therein. Theoretical studies are relatively few; see [5] for MPA in linear cellular systems and [6] for systems with uniform traffic. In [1], an upper bound on the carried traffic per channel achievable in a cellular system by any channel assignment algorithm has been derived. This bound is asymptotically achievable by an FCAA, in the limit of an infinite number of available channels, under the assumption of a fixed traffic pattern. In this paper, we attempt to analyze MPA for general cellular systems with arbitrary traffic patterns, in terms of the blocking probability and the carried traffic.

We use the hypergraph model for the cellular network as in [1]. This model is paraphrased in Section II for convenience. This section also addresses the stochastic properties of the model and the state space of the system. MPA must honor a call when the resulting state is in the state space. In Section III, we compute the asymptotic performance of MPA using a nonlinear optimization problem and compare it with the (asymptotically achievable) bound derived in [1] Asymptotic results give us insight into the behavior of MPA and using asymptotic properties we approximate the cellular system by a multirate fixed routing loss network in Section IV and show that for practical system sizes, this approximation works well. In Section V, we compare MPA with the optimal FCAA of [1], and the DCAA, and observe that MPA works best in low and moderate traffic. But in the heavy traffic case, its performance is worse than that of the other algorithms.

Implementation of MPA in an arbitrary two-dimensional (2-D) cellular system is computationally hard. Even to find, whether a state is admissible under MPA, is an NP complete $^{1}$ problem. MPA requires centralized control of channel assignment and to accommodate a new call, network wide channel rearrangement may be required. Moreover the number of calls to which channels may have to be reassigned grows without limit as the system size increases. In Section VI, we attempt to give a good procedure to implement MPA, and suggest approximate real time algorithms. We also consider the question of the number of reassignments in the worst and average cases.

\footnotetext{
${ }^{1}$ In Section II, we show that this problem is analogous to a hypergraph multicoloring problem, and an integer linear program has to be solved for this.
}

Mathematical analysis of cellular systems with handoff is inherently difficult, as stochastic properties of the cells are not independent. We make the simplifying assumption that there are no handoffs in the system.

The contribution of this paper can be summarized in the following points.

1) It provides a mathematical tool to derive the asymptotic performance of MPA in a general cellular network.

2) It provides a simple approximation to the performance of MPA in the finite channel case.

3) It suggests a good procedure to implement this complex algorithm in real time.

\section{Cellular Network Model}

\section{A. Cellular Model and Offered Traffic}

We assume our cellular system to be a finite set of $N$ nonoverlapping cells and assume that the underlying offered traffic model is known for each cell, and is independent from cell to cell. These $N$ cells share a common set of $n$ channels, and the intensity of offered traffic is measured in Erlangs per channel. Thus, if $\nu_{i}$ denotes the expected number of calls that would be in progress in cell $i$ at a given time, if all call requests in that cell could be honored, then the intensity of the offered traffic in cell $i$ is $r_{i}=\nu_{i} / n$ Erlangs per channel. The overall traffic in the system is then $\nu=\sum_{i} \nu_{i}$ Erlangs and the overall intensity of offered traffic is $r=\nu / n$ Erlangs per channel. The ratio $p_{i}=\nu_{i} / \nu$ represents the fraction of total traffic offered in cell $i$, and we call the vector $p=\left(p_{1}, p_{2}, \cdots, p_{N}\right)$ the traffic pattern. We shall assume the traffic pattern to be fixed in our analysis.

\section{B. Channel Assignment Model}

A call request in a particular cell is either assigned to one of the $n$ channels or blocked by a channel assignment algorithm. A blocked call disappears from the system. The channels assigned to calls must satisfy certain channel reuse constraints. There is a fixed collection $E=\left\{E_{1}, E_{2}, \cdots, E_{k}\right\}$ of subsets of cells, called forbidden subsets. A channel cannot be used simultaneously in each cell of a forbidden set. The assumption of fixed forbidden sets means that we are restricting our attention to channel assignment algorithms which make decisions based only on the knowledge of the cells in which the calls arise.

A finite set together with a collection of subsets is called a hypergraph, and so we view our cellular system as a hypergraph system denoted by $H$. Cells form the set of vertices, $\left\{v_{1}, v_{2}, \cdots, v_{N}\right\}$, where vertex $v_{i}$ corresponds to cell $i$, and forbidden sets form the set of edges of $H$. The traditional cellular system model based on minimum reuse distance, in which a channel can be used in two cells at same time provided these cells are sufficiently separated in space, is encompassed in our generalized hypergraph model with edges being sets of two cells if and only if they are not separated by the reuse distance.

An independent set of a hypergraph $H$ is a set of vertices which contains no edge as a subset. A maximal independent 


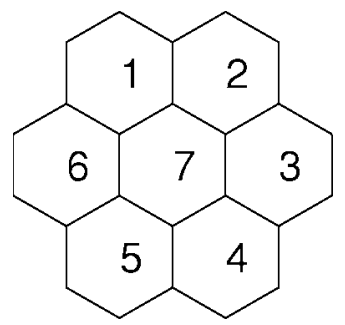

Fig. 2. The symmetrical seven-cell system.

set (MIS) is an independent set which is not a proper subset of any other independent set. In the cellular network context, an independent set denotes the set of cells, in which a channel can be used simultaneously. If the channel is in use in all the cells of an MIS, no other cell can use it. We assume $H$ has $M$ maximal independent sets $\left\{V_{1}, V_{2}, \cdots, V_{M}\right\}$. We define the incidence matrix $A=\left(a_{i j}\right)$ as

$$
a_{i j}= \begin{cases}1, & \text { if } v_{i} \in V_{j} \\ 0, & \text { if } v_{i} \notin V_{j} .\end{cases}
$$

The hypergraph can be reconstructed from the incidence matrix $A$ and we prefer to represent the cellular system by $A$.

For any channel assignment algorithm at any point in time, the sets of cells using a particular channel must be a subset of an MIS of $H$.

Example 2: Fig. 2 shows the symmetrical seven-cell system. The pairs of all adjacent cells and the sets $\{1,3,5\}$ and $\{2,4,6\}$ are assumed to constitute edge set of the hypergraph representing this system. The MIS's for this system are $\{1$, $3\},\{1,4\},\{1,5\},\{2,4\},\{2,5\},\{2,6\},\{3,5\},\{3,6\},\{4$, $6\}$, and $\{7\}$.

\section{State Space in Maximum Packing}

The state space of the system and the stochastic properties of the arrival and departure processes completely define the system for MPA. A new call must be honored if and only if the resulting state is in the state space. The real advantage of MPA from an analytic point of view is that a simple state space results; it does not require keeping track of which channel is in use in which cell. Thus, knowing the stationary probability distribution of the states in the state space for the given call arrival and departure processes, we can find the carried traffic and the blocking probabilities in the cells. However we will see later that this solution is not practical because the state space is very large.

1) Finite Channel State Space: We define the state of the system as an integer vector $\mathbf{z}=\left(z_{1}, z_{2}, \cdots, z_{N}\right)$ of length $N$, where $z_{i}$ is the number of active calls in cell $i$. The channel reuse constraints impose restriction upon the values that the state $\mathbf{z}$ can take, and hence define a state space $\mathcal{S}(n)$ for a given channel assignment strategy, which, of course, depends on the total number of available channels $n$.

MPA admits a call if and only if the resulting state would be in the state space $\mathcal{S}_{\mathrm{MP}}(n)$. Also we assume call terminations are not blocked, i.e., if $\mathbf{z} \in \mathcal{S}(n)$ and $z_{i} \geq 1$ then $\left(z_{1}, \cdots, z_{i}-\right.$ $\left.1, \cdots, z_{N}\right) \in \mathcal{S}(n)$. This is called the coordinate convex property.
Theorem 1: A call vector $\mathbf{z}$ is admissible under MPA if and only if there exists a nonnegative $M$-dimensional integer vector $\mathbf{Z}$, such that

$$
\begin{aligned}
& \sum_{j=1}^{M} a_{i j} Z_{j} \geq z_{i}, \quad i=1, \cdots, N \\
& \sum_{j=1}^{M} Z_{j} \leq n, \quad \text { where } \mathbf{z} \in \mathcal{Z}_{+}^{N} \text { and } \mathbf{Z} \in \mathcal{Z}_{+}^{M} .
\end{aligned}
$$

Here $\mathcal{Z}_{+}^{N}$ (resp. $\mathcal{Z}_{+}^{M}$ ) is the set of nonnegative integer vectors of length $N$ (resp. $M$ ).

Proof: If an integer vector $\mathbf{Z}$ satisfying these conditions exists, by allocating $Z_{j}$ channels to all cells of the $j$ th MIS, cell $i$ can support $\sum_{j=1}^{M} a_{i j} Z_{j}$ calls; since $z_{i} \leq \sum_{j} a_{i j} Z_{j}$, it can support $z_{i}$ calls too.

Now if some channel allocation supporting the call vector exists then the set of cells to which each channel is assigned must be a subset of at least one of the MIS's. Counting it in one (and only one) of them, we can form the vector $\mathbf{Z}$, which satisfies inequalities 1 and 2 .

Thus, the state space of MPA, with $n$ available channels, can be written as

$$
\begin{aligned}
& \mathcal{S}_{\mathrm{MP}}(n) \\
& =\left\{\mathbf{z} \in \mathcal{Z}_{+}^{N}: \exists \mathbf{Z} \in \mathcal{Z}_{+}^{M}, \quad \text { such that } A \mathbf{Z} \geq \mathbf{z}, \sum_{j=1}^{M} Z_{j} \leq n\right\} .
\end{aligned}
$$

a) Virtual channel assignment: Theorem 1 shows that a call vector $\mathbf{z}$ is admissible under MPA if and only if there exists an $M$-dimensional integer vector $\mathbf{Z}=\left(Z_{1}, \cdots, Z_{M}\right)$, satisfying constraints (1) and (2). We notice that if $Z_{j}$ channels are assigned in all cells of the $j$ th MIS then the total number of available channels in the $i$ th cell is $\sum_{j} a_{i j} Z_{j}$, which being greater than $z_{i}$, can support $z_{i}$ calls. Thus, we say that an MIS is virtually assigned a channel, even though only a subset of this MIS is actually using it. Let a virtual assignment vector $\mathbf{Z}$ exist satisfying ${ }^{2}$ the state vector $\mathbf{z}$. Then if an incoming call results in a state, which is still satisfied by this assignment, no ongoing call needs to be reassigned a channel.

2) Asymptotic State Space: We now introduce an asymptotic state space for the cellular network. We consider a sequence of traffic models, where the traffic pattern $p$, and offered traffic intensities $r_{i}$ remain fixed and the number of channels, $n$, increases. Thus, the offered traffics $\nu_{i}$ increase linearly with $n$. We define the normalized state vector $\mathrm{x} \in \mathcal{R}_{+}^{N}$, in the asymptotic limit, to be

$$
\mathrm{x}=\lim _{n \rightarrow \infty} \frac{1}{n} \mathbf{z}, \quad \text { where } \mathrm{x} \in \mathcal{R}_{+}^{N} .
$$

Here $\mathcal{R}_{+}^{N}$ is the set of nonnegative real vectors of length $N$.

Let $\mathrm{x}=\left\{x_{1}, x_{2}, \cdots, x_{N}\right\}$. Note that $x_{i}$ denotes the number of calls carried per channel in cell $i$.

\footnotetext{
${ }^{2}$ We say that a vector $\mathbf{Z} \in \mathcal{Z}_{+}^{M}$ satisfies state $\mathbf{z} \in \mathcal{Z}_{+}^{N}$, if it satisfies inequalities (1) and (2).
} 
The state space in the asymptotic limit is thus given by

$$
\begin{aligned}
& \mathcal{S}_{\mathrm{MP}}= \\
& \cdot\left\{\mathrm{x} \in \mathcal{R}_{+}^{N}: \exists \mathbf{X} \in \mathcal{R}_{+}^{M}, \text { such that } A \mathbf{X} \geq \mathrm{x}, \sum_{j=1}^{M} X_{j} \leq 1\right\} .
\end{aligned}
$$

Note that $\mathbf{X}$ and $\mathbf{X}$ are real vectors in contrast to $\mathbf{z}$ and $\mathbf{Z}$ in Theorem 1 which were integer vectors.

The set $\mathcal{F}$ of all feasible vectors $\mathbf{X}$ is bounded and is the convex hull of a finite number of extreme points, which are the origin and the unit vector $\mathbf{e}_{j}, j=1, \cdots, M$ in the $j$ th direction. Hence, $A \mathcal{F}$ is also a convex hull of a finite number of extreme points $\mathbf{a}_{j}$, where $\mathbf{a}_{j}$ is $j$ th column of matrix $A$. This observation combined with the following properties of state space: 1) nonnegativity $x_{i} \geq 0$ and 2) coordinate convexity $\mathrm{x} \in \mathcal{S}, \mathrm{x}^{\prime} \leq \mathrm{x} \Rightarrow \mathrm{x}^{\prime} \in \mathcal{S}$ shows that $\mathcal{S}_{\mathrm{MP}}$ is the convex hull of points $\mathbf{a}_{j}^{\prime}$, where $\mathbf{a}_{j}^{\prime}$ is a $(0,1)$ vector in $\mathcal{R}_{+}^{N}$ corresponding to an independent set (and not just the maximal ones).

From this explicit form, which requires the list of all extreme points, i.e., all independent sets of the system, we can derive a system of linear inequalities of the form $\bar{A} \mathbf{x} \leq \bar{b}$ representing the convex polyhedron $\mathcal{S}_{\mathrm{MP}}$.

Example 3: The linear three-cell system is shown in Fig. 1. The minimum forbidden sets for this system are $\{1,2\}$ and $\{2,3\}$ and from this we derive the MIS to be $\{1,3\}$ and $\{2\}$. The state space for this system, is the convex hull of the points $(0,0,0),(1,0,0),(0,0,1),(1,0,1)$, and $(0,1,0)$. The state space satisfies the following set of inequalities:

and

$$
x_{1}+x_{2} \leq 1, \quad x_{2}+x_{3} \leq 1
$$

$$
0 \leq x_{i} \leq 1, \quad \text { for } i=1,2,3 .
$$

The implicit form for the real state space can be approximately ${ }^{3}$ extended to the integer state space $\mathcal{S}_{\mathrm{MP}}(n)$ as

$$
\mathcal{S}_{\mathrm{MP}}(n) \approx n \mathcal{S}_{\mathrm{MP}} \cap \mathcal{Z}_{+}^{N}
$$

Though this form provides a quick check as to whether a call is admissible in the system or not, it does not give any insight into the actual channel allocation and the calls to be repacked to accommodate a new call. Hence, we shall continue using the explicit form, i.e., checking for the possibility of a vector $\mathbf{Z}$ or $\mathbf{X}$ for a call vector, in the rest of our analysis.

\section{Stochastic Properties of the Model}

Having specified the state space of the system, we now characterize the stochastic properties of the model.

Let the state of the system at time $t$ be $\mathbf{z}(t)=\left(z_{i}(t)\right.$; $i=1, \cdots, N)$. Then if we assume that the call arrivals are Poisson and the call holding times are exponential, the stochastic process $\{\mathbf{z}(t), t \geq 0\}$ can be considered as a Markov chain on the state space $\mathcal{S}_{\mathrm{MP}}(n)$. With our assumption of intercell independence, this Markov chain is time reversible

\footnotetext{
${ }^{3}$ Actually $\mathcal{S}_{\mathrm{MP}}(n) \subseteq n \mathcal{S}_{\mathrm{MP}} \cap \mathcal{Z}_{+}^{N}$. For some vector $\mathbf{z}$, if $\bar{A} \mathbf{z} \leq n \bar{b}$, then a real vector $\mathbf{Z} \in \Re_{+}^{N}$ exists, satisfying (1) and (2), but such an integer vector may not exist.
}

with the unique stationary distribution $\pi(\mathbf{z})=P\{\mathbf{z}(t)=\mathbf{z}\}$ given by (see [7] and [8])

$$
\pi(\mathbf{z})=G^{-1} \prod_{i=1}^{N} \frac{\nu_{i}^{z_{i}}}{z_{i} !}, \quad \mathbf{z} \in \mathcal{S}_{\mathrm{MP}}
$$

where $\nu_{i}=r n p_{i}$, is the offered traffic in cell $i$, and $G$ is the normalizing constant

$$
G=\sum_{\mathbf{z} \in \mathcal{S}_{\mathrm{MP}}} \prod_{i=1}^{N} \frac{\nu_{i}^{z_{i}}}{z_{i} !} .
$$

Using the hypergraph model for cellular networks, we have derived the form of the state space, which is analogous to that of a fixed-routing loss network. Furthermore, the similarity of the stochastic properties of arrival and departure processes in the two kinds of networks enables us to use the well-known results from the field of loss networks. ${ }^{4}$

Though the hypergraph model provides us the instrument to study the channel assignment problem in cellular networks mathematically, the complexity involved is very high. An algorithm for the generation of all the MIS's of a hypergraph is described in [9]. The number of MIS's $(M)$ grows exponentially with the system size $(N)$. So its application is limited to small systems.

\section{ASYMPTOTIC ANALYSIS}

In this section, we shall study the performance of the maximum packing algorithm for a large number of available channels. We shall refer to this as the asymptotic performance. We shall assume a fixed traffic pattern and a given offered traffic intensity.

\section{A. Formulation of Primal Problem}

The problem of finding the carried traffic when call admission depends only on whether the resulting state is in the state space and the state has a probability distribution of the type given in (6), has been well addressed in the literature on communication networks theory.

In our analysis of MPA in the asymptotic case, we use the following result due to Kelly [7]. Let $\overline{\mathrm{x}} \in \Re_{+}^{N}$ be the unique solution of the following optimization problem:

$$
\max \sum_{i=1}^{N}\left[x_{i}+x_{i} \log \frac{r_{i}}{x_{i}}\right], \quad \text { subject to } \mathrm{x} \in \mathcal{S} .
$$

Then

$$
\lim _{n \rightarrow \infty} \frac{1}{n} E\left[z_{i}(n)\right] \rightarrow \bar{x}_{i}, \quad i=1, \cdots, N .
$$

We extend this result to cellular networks with the modifications required due to the much more complicated state space. Using the form (4) for the state space and by introducing $N$ slack variables $X_{M+1}, \cdots, X_{M+N}$, for the inequalities, we form the following optimization problem, whose maximizing

\footnotetext{
${ }^{4}$ Analogies between cellular and loss networks have been made by other authors [2], [7]. However, in these papers, the reuse model for the cellular network was not as general as in this paper. They used a graph model which is a special case of our hypergraph model.
} 
value $\overline{\mathrm{x}}$ gives the carried traffic per channel in the asymptotic case.

1) Primal Optimization Problem:

$$
\max \sum_{i=1}^{N}\left[x_{i}+x_{i} \log \frac{r_{i}}{x_{i}}\right]
$$

subject to

$$
\begin{gathered}
x_{i} \geq 0, \quad i=1, \cdots, N \\
X_{j} \geq 0, \quad j=1, \cdots, M+N \\
\quad \sum_{j=1}^{M} X_{j} \leq 1
\end{gathered}
$$

where

$$
x_{i}=\sum_{j=1}^{M} a_{i j} X_{j}-X_{i+M}, \quad i=1, \cdots, N .
$$

We view the $x_{i}$ 's as functions of the augmented vector $\tilde{\mathbf{X}}=\left(X_{1}, \cdots, X_{M}, X_{M+1}, \cdots, X_{M+N}\right)$. The objective function in this problem is strictly convex $\cap$, and it tends to $-\infty$, as $x_{i} \rightarrow \infty$. Also from the intrinsic form of $\mathcal{S}_{\mathrm{MP}}$ (see Section II-C), the feasible region is convex. Thus, there exists a unique solution to the primal optimization problem for $\overline{\mathrm{x}}$.

Thus, we have formed a nonlinear optimization problem in $M+N$ variables and $M+N+1$ inequalities. By solving this we can find the vector $\overline{\mathbf{X}}$, which maximizes this and hence can find the unique carried traffic vector $\bar{x}$ and from it we can derive the blocking probabilities in different cells.

\section{B. Derivation of Dual Problem}

Consider the Lagrangian form for the optimization problem (9)

$$
\begin{aligned}
L(X, y, \pi, \lambda)= & -\sum_{i=1}^{N}\left(x_{i}+x_{i} \log \frac{r_{i}}{x_{i}}\right)-y\left(1-\sum_{j=1}^{M} X_{j}\right) \\
& -\sum_{j=1}^{M+N} \lambda_{j} X_{j}-\sum_{i=1}^{N} \pi_{i} x_{i}
\end{aligned}
$$

where $\pi_{i} \geq 0, \lambda_{j} \geq 0$, and $y \geq 0$ are the dual variables corresponding to inequalities (10)-(12), respectively. Hence, the dual slackness conditions are

$$
\begin{gathered}
\pi_{i} x_{i}=0, \quad i=1, \cdots, N \\
\lambda_{j} X_{j}=0, \quad j=1, \cdots, M+N \\
y\left(1-\sum_{j=0}^{M} X_{j}\right)=0 .
\end{gathered}
$$

The dual problem can be formed by maximization of this Lagrange form, with constraints

$$
\nabla_{X} L=0 \Rightarrow \frac{\partial}{\partial X_{j}} L=0, \quad j=1, \cdots, M+N
$$

which results in

$$
y-\lambda_{j}-\sum_{i=1}^{N} a_{i j}\left[\pi_{i}+\log \left(r_{i} / x_{i}\right)\right]=0, \quad j=1, \cdots, M
$$

and

$$
\lambda_{i+M}-\left[\pi_{i}+\log \left(r_{i} / x_{i}\right)\right]=0, \quad i=1, \cdots, N
$$

Plugging back the values of $\lambda$ 's into the Lagrangian (14), we get

$$
L(x, y, \lambda, \pi)=-\sum_{i=1}^{N} x_{i}-y
$$

Thus, the dual optimization problem is

$$
\max -\sum_{i=1}^{N} x_{i}-y
$$

where $x_{i} \geq 0, \pi_{i} \geq 0$ for $i=1, \cdots, N$, and constraints $\lambda_{j} \geq 0$ result in

$$
\begin{gathered}
y \geq \sum_{i+1}^{N} a_{i j}\left[\pi_{i}+\log \left(\frac{r_{i}}{x_{i}}\right)\right], \quad j=1, \cdots, M \\
\pi_{i}+\log \left(\frac{r_{i}}{x_{i}}\right) \geq 0, \quad i=1, \cdots, N .
\end{gathered}
$$

Now we observe that if the optimum $x_{i}=0$, for any $i$, when $r_{i}$ has some finite value, then the condition (21) results in $y \geq \infty$ in (20), and then the maximizer solution occurs at $-\infty$, which is a contradiction. Hence, in the maximum packing algorithm no optimum $x_{i}$ can be zero if $r_{i}>0$. The dual slackness conditions (15) imply that if $x_{i} \neq 0$, then $\pi_{i}=0$ for $i=1, \cdots, N$.

The conditions (22) now become

$$
\log \frac{r_{i}}{x_{i}} \geq 0 \Rightarrow x_{i} \leq r_{i}, \quad i=1, \cdots, N
$$

This is intuitively satisfactory as the carried traffic can never be greater than the offered traffic.

Now we define

$$
q_{i}=\log \frac{r_{i}}{x_{i}}, \quad \text { so that } x_{i}=r_{i} e^{-q_{i}}, \quad i=1, \cdots, N \text {. }
$$

Constraints $x_{i} \geq 0$ imply a one-to-one correspondence between the $q_{i}$ and the $x_{i}$, and the condition $x_{i} \leq r_{i}$ gives $q_{i} \geq 0$. We denote the blocking probability in the $i$ th cell by $B_{i}$. Then $q_{i}$ can be written in terms of $B_{i}$ as

$$
e^{-q_{i}}=\frac{x_{i}}{r_{i}}=1-B_{i}, \quad B_{i} \in[0,1), i=1, \cdots, N
$$

The dual problem can be written in terms of $q_{i}$ s and $y$ as follows. 


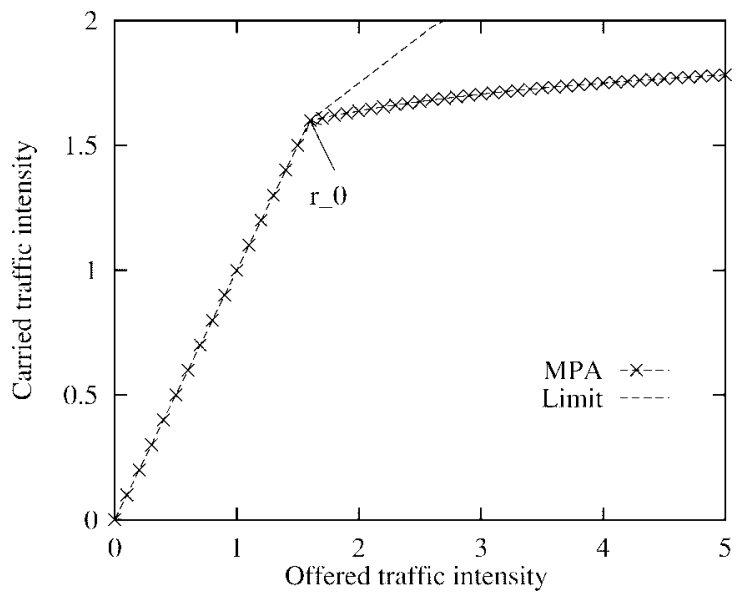

Fig. 3. Asymptotic performance of MPA in the seven-cell system compared to the performance limit. $r_{0}$ is the capacity of the cellular system and will be discussed later.

\section{2) Dual Optimization Problem:}

$$
\begin{aligned}
\text { Minimize } & \sum_{i=1}^{N} r_{i} e^{-q_{i}}+y \\
\text { subject to } & y \geq \sum_{i=1}^{N} a_{i j} q_{i}, \quad j=1, \cdots, M \\
\text { and } \quad & q_{i} \geq 0, \quad i=1, \cdots, N .
\end{aligned}
$$

Thus, we have reduced the primal problem of $M+N$ variables and $M+N+1$ constraints with a complex objective function to a comparatively easy problem of $N+1$ variables and $M+N$ constraints. In terms of computational complexity this dual problem is preferred to evaluate the asymptotic performance of MPA in cellular networks.

Example 4: Consider the symmetrical seven-cell system shown in Fig. 2. Let the traffic pattern be $\mathbf{p}=\left(\frac{1}{8}, \frac{1}{8}, \frac{1}{8}, \frac{1}{8}, \frac{1}{8}\right.$, $\left.\frac{1}{8}, \frac{1}{4}\right)$. We solve the dual optimization problem for this system. By making use of the symmetry of the problem, inequalities (26) reduce to $q_{i}=y / 2, i=1, \cdots, 6$ and $q_{7}=y$. Thus, the objective function (25) becomes a function of the single variable $y$ and is minimized when

$$
y=\max \left(0,-2 \log \frac{\sqrt{9+64 / r}-3}{4}\right) .
$$

Thus, the blocking probability in all cells can be calculated using (24) and the carried traffic intensity can be determined. In Fig. 3, this carried traffic intensity is compared with the performance limit for the system derived in [1].

Example 5: We now consider the 16-cell system shown in Fig. 4, with uniform traffic. The system has 82 MIS's, 45 of cardinality 4,36 of cardinality 5 , and 1 of cardinality 6. We find the carried traffic intensity in MPA using the dual optimization problem and compare the results with the performance limit in Fig. 5.

Theorem 2: The blocking probabilities $B_{i} \in[0,1), i=$ $1, \cdots, N$ are solutions of the following equations, for some

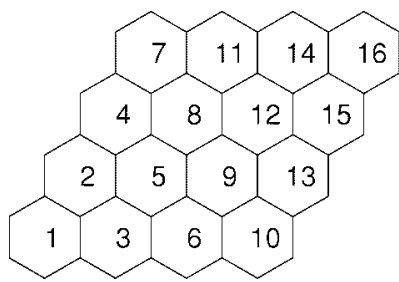

Fig. 4. The 16-cell system.

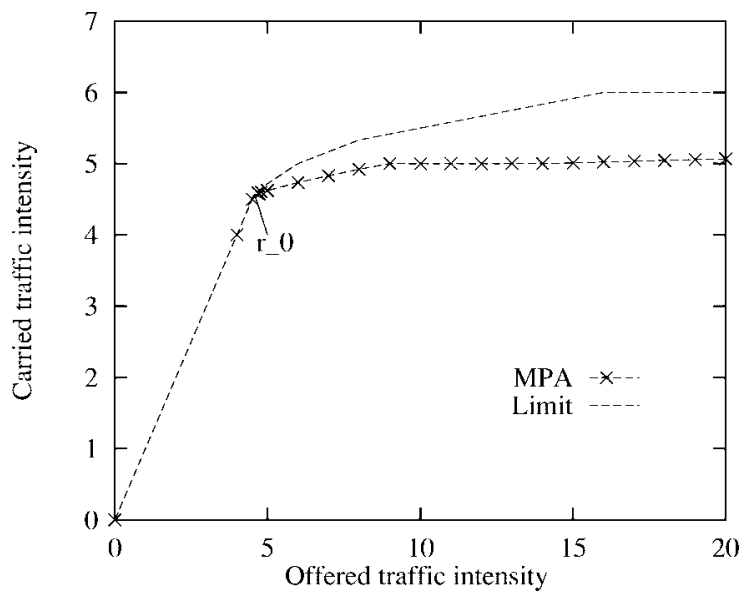

Fig. 5. Asymptotic performance of MPA in the 16-cell system compared to the performance limit. $r_{0}$ is the capacity of the cellular system and will be discussed later.

$\mathbf{X}$ and $y$ :

$$
\begin{aligned}
\sum_{j=1}^{M} a_{i j} X_{j} & =r p_{i}\left(1-B_{i}\right), \quad \text { if } B_{i}>0 \\
& \geq r p_{i}\left(1-B_{i}\right), \quad \text { if } B_{i}=0 \\
\prod_{i=1}^{N}\left(1-B_{i}\right)^{a_{i j}} & =\exp (-y), \quad \text { if } X_{j}>0 \\
& \geq \exp (-y), \quad \text { if } X_{j}=0 \\
\sum_{j=1}^{M} X_{j} & =1, \quad \text { if } y>0 \\
& \leq 1, \quad \text { if } y=0 .
\end{aligned}
$$

Proof: From (19) and (23) $\lambda_{i+M}=\log \left(r_{i} / x_{i}\right)=q_{i}$, $i=1, \cdots, N$. Now using (24), which imply $q_{i}=0$ if and only if $B_{i}=0$, (13) and (16), we get the first set of equations.

$$
\begin{aligned}
& X_{i+M}=0, \quad \text { if } q_{i}>0 \\
& \geq 0, \quad \text { if } q_{i}=0
\end{aligned}
$$

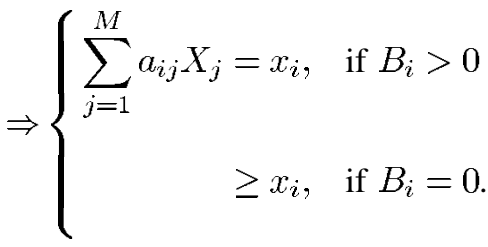

Now note that $X_{j}$ is the dual variable of the $j$ th inequality in (26). Multiplying by -1 and taking the exponential of both the sides results in the second set of equations. 
The last equation follows directly from the dual slackness condition (17).

$X_{j}$ can be interpreted as the fraction of the total channels assigned to the $j$ th MIS. Then, Theorem 2 can be interpreted as follows.

1) If a cell has positive blocking, the carried traffic equals the channel allocation.

2) For a large number of channels available, blocking events in different cells may be assumed independent. Then $\prod_{i}\left(1-B_{i}\right)^{a_{i j}}$ denotes the probability of finding at least one free channel in all the cells of MIS $j$. Thus, we can say that MPA assigns channels in such a manner that all MIS's that have a channel assigned to them have the same probability of having a free channel. Moreover, the probability of finding a free channel in all the cells of an MIS that is not assigned any channel, is larger.

3) All channels are assigned, if there is blocking in any cell.

Consider an MIS which is assigned a channel. If a channel can be assigned to new calls in all the cells of this MIS, a free channel can be found (with repacking, if necessary) in the system. Thus, from (28) the value of $\exp (-y)$ in the (optimal) solution can be interpreted as the probability of finding at least one channel free in the system, in the asymptotic limit. Thus, for a given offered traffic intensity vector, we can write

$$
\lim _{n \rightarrow \infty} \frac{P\left[\mathbf{z} \in \mathcal{S}_{\mathrm{MP}}(n-1)\right]}{P\left[\mathbf{z} \in \mathcal{S}_{\mathrm{MP}}(n)\right]}=e^{-y} .
$$

Example 6: We apply Theorem 2 to evaluate the asymptotic performance of MPA in the linear 3 cell network shown in Fig. 1 with uniform traffic. Both MIS's $\{1,3\}$ and $\{2\}$ must be active to prevent complete blocking in any cell and by symmetry $B_{1}=B_{3}$, hence

$$
\left(1-B_{1}\right)\left(1-B_{3}\right)=\left(1-B_{2}\right)=\left(1-B_{1}\right)^{2} \text {. }
$$

Thus

$$
1 \geq X_{1}+X_{2} \geq \frac{r}{3}\left(1-B_{1}\right)\left(2-B_{1}\right)
$$

Hence, for $r \leq \frac{3}{2}, B_{1}=0$ and hence $B_{2}=0$. For $r>\frac{3}{2}$, (31) must be satisfied with equality. Hence

$$
B_{1}=B_{3}=\frac{1}{2}\left(3-\sqrt{1+\frac{12}{r}}\right)
$$

and

$$
B_{2}=\frac{1}{2}\left(1-\frac{6}{r}+\sqrt{1+\frac{12}{r}}\right) .
$$

We define the carried traffic function $T_{\mathrm{MP}}(r)$ for the MPA to be the expected number of calls carried in the system per channel. Thus, if $q_{i}$ 's are the solution to the dual optimization problem

$$
\begin{aligned}
T_{\mathrm{MP}}(r) & =r \sum_{i=1}^{N} p_{i} \exp \left(-q_{i}\right) \\
& =\sum_{i=1}^{N} r p_{i}\left(1-B_{i}\right) .
\end{aligned}
$$

The carried traffic function calculated using the above formula for the system considered in Example III-B is

$$
T_{\mathrm{M \Gamma}}(r)=\min \left(r, 1+\frac{r}{6}\left[\sqrt{1+\frac{12}{r}}-1\right]\right) \text {. }
$$

Theorem 3: $T_{\mathrm{MP}}(r)=r$ for all $r \leq r_{0}$, where $r_{0}$ is the value of the following linear program:

$$
\begin{array}{ll}
\operatorname{maximize} & r \\
\text { subject to } & X_{j} \geq 0, \quad \text { for } j=1, \cdots, N \\
& \sum_{j=1}^{M} X_{j} \leq 1 \\
& \sum_{j=1}^{M} a_{i j} X_{j} \geq r p_{i}, \quad \text { for } j=1, \cdots, N .
\end{array}
$$

Proof: Using the Lagrangian method, we can derive the dual of the dual optimization problem as

$$
\begin{array}{ll}
\operatorname{maximize} & 2 r \sum_{i=1}^{N} p_{i} e^{-q_{i}} \\
\text { subject to } \quad & X_{j} \geq 0, \quad q_{i} \geq 0, \\
& \sum_{j=1}^{M} X_{j} \leq 1 \\
& \sum_{j=1}^{M} a_{i j} X_{j} \geq r p_{i} e^{-q_{i}} .
\end{array}
$$

To maximize the objective function in this problem, $q_{i}$ 's must be as small as possible. Now for any $r<r_{0}$, let the solution of the LP considered in the theorem occurs at $\bar{X}$. Then $\overline{\mathbf{X}}$ and $q_{i}=0, i=1, \cdots, N$ are in the feasible region of this dual of the dual problem, and maximize the objective function. Thus, at the solution of this dual of the dual problem, and hence the original dual problem, $q_{i}=0, i=1, \cdots, N$. Hence, at this $r, B_{i}=0, i=1, \cdots, N$ and $T_{\mathrm{MP}}(r)=r$.

Remark: In [1], it is shown that $r_{0}$, which is the solution of same linear program can be interpreted as capacity of the cellular network. If the offered traffic $r$ exceeds $r_{0}$, then for any channel assignment algorithm, a positive fraction of all call requests must be blocked, whereas if the offered traffic is less than $r_{0}$, all call requests can be honored, if the number of channels is sufficiently large. Thus, Theorem 3 shows that asymptotically MPA tracks the limiting performance for $r<r_{0}$. Though disappointingly for $r>r_{0}$, the performance is very poor (see, for example, Figs. 3 and 5).

The asymptotic analysis provides us the intuitive perception of the behavior of MPA performance for finite number of channels in the system. In the next section, using the results in Theorem 2, we approximate the cellular network by a simple fixed-routing loss network. We shall see that MPA converges rapidly to its asymptotic limit, compared to other algorithms and hence for a finite number of channels in the system, its performance is better up to the point $r_{0}$. 


\section{Finite Channel ApProximation}

While asymptotic analysis gives a feel about the performance of an algorithm, it is also necessary to analyze the performance in practical systems with a finite number of channels $(n<\infty)$. Exact analysis of the MPA is possible, in principle, as we know the state space and the probability distribution of the states, and the (average) carried traffic is just the expected number of the calls in progress. The blocking probability can also be found exactly, in principle.

We define the critical set $\mathcal{O}_{i}(n), i=1, \cdots, N$, corresponding to each cell, as

$$
\mathcal{O}_{i}(n)=\left\{\mathbf{z :} \quad \mathbf{z} \in \mathcal{S}_{M P}(n), \mathbf{z}+\mathbf{e}_{i} \notin \mathcal{S}_{\mathrm{MP}}(n)\right\}
$$

where $\mathbf{e}_{i}$ is the unit vector in the $i$ direction and thus denotes an additional call in cell $i$.

The blocking probability in cell $k$ is given by

$$
B_{k}=P\left(\mathbf{z} \in \mathcal{O}_{k}\right)=\frac{\sum_{\mathbf{z} \in \mathcal{O}_{k}(n)} \prod_{i=1}^{N} r_{i}^{z_{i}} / z_{i} !}{\sum_{\mathbf{z} \in \mathcal{S}_{\mathrm{MP}}(n)} \prod_{i=1}^{N} r_{i}^{z_{i}} / z_{i} !} .
$$

This simple form may provide a complete solution for small networks. But the state of the system grows exponentially with the number of cells $N$ and polynomially with the number of channels. Hence, it is impractical to use the direct form for all but very small networks. This is the motivation for the approximation we shall discuss in this section.

Consider the dual optimization problem (25), in the asymptotic case. The problem can be rewritten by setting $q_{i}=b_{i} y$, $b_{i} \geq 0, i=1, \cdots, N$. Here the $b_{i}$ 's can be interpreted as weighting variables. We can now rewrite (24) as

$$
1-B_{i}=e^{-q_{i}}=\left(e^{-y}\right)^{b_{i}}, \quad i=1, \cdots, N .
$$

In Section III, we noted that $e^{-y}$ can be interpreted as the probability of finding at least one free channel in the system in the asymptotic limit. Thus, $\left(e^{-y}\right)^{b_{i}}$ denotes the probability of finding at least $b_{i}$ channels free in the system, ${ }^{5}$ in the asymptotic limit. Thus, knowing $b_{i}$, we can approximate the blocking probabilities in the cellular network by those of a link with capacity $n$, which supports $N$ classes of connections. Connections belonging to class $i$ require $b_{i}$ circuits from the link and have an offered rate $\nu_{i}=r n p_{i}$.

${ }^{5}$ Let $L_{r}$ denotes the stationary probability that a call requesting route $r$ is lost in a loss network. In [8], Kelly proves that in the asymptotic limit

$$
1-L_{r} \rightarrow \prod_{j}\left(1-B_{j}\right)^{A_{j r}}
$$

where $B_{j} \in[0,1)$ is a parameter associated with the link $j . B_{j}$ may be interpreted as the probability that the link $j$ is full.

With only one link this becomes

$$
1-L_{r} \rightarrow\left(1-B_{1}\right)^{A_{1 r}}
$$

In our system, there is only one link. The route index is replaced by $i$ and $A_{1 r}$ by $b_{i}$.

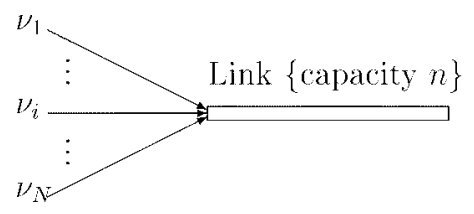

Fig. 6. The single-link approximation.

\section{A. Single-Link Approximation}

Thus, the approximate analogous communication system is as shown in Fig. 6 , and the $b_{i}$ must satisfy $b_{i} \geq 0$, $i=1, \cdots, N$, and

$$
\sum_{i=1}^{N} b_{i} z_{i} \leq n
$$

In this network, the blocking probability for a class $i$ connection is exactly given by (see [10] and [11])

$$
B^{(i)}\left[n ; \nu_{k}, k=1, \cdots, N\right]=\sum_{j=0}^{b_{i}-1} q(n-j), \quad i=1, \cdots, N
$$

where $q(j)$ is the solution of following recursive equation:

$$
q(j)=\frac{1}{j} \sum_{i=1}^{N} \nu_{i} b_{i} q\left(j-b_{i}\right), \quad j=\min _{i} b_{i}, \cdots, n
$$

with $q(j)=0$ for $j<0,1 \leq j<\min _{i} b_{i}$ and

$$
\sum_{j=0}^{n} q(j)=1
$$

$q(j)$ can be interpreted as the probability that exactly $j$ circuits on the link are in use. Then the recursive equation looks like the balance equation for a one-dimensional (1-D) Markov chain.

From this, we determine the exact blocking probability for class $i$ connections and use it as an approximation to the blocking probability in cell $i$ in the cellular case. Note that the $b_{i}$ 's are determined from the solution to the dual optimization problem by setting $q_{i}=b_{i} y$. For the case $r \leq r_{0}$, when $y=0$ and all $q_{i}=0$, we take the values $b_{i}$ 's, at the point $r=r_{0}+\epsilon$, where $\epsilon$ is a small positive value. The problem that remains is that $b_{i}$ may be, and in general is, a fractional value. We resolve this problem by multiplying (35) by a suitable integer constant, which makes all the $b_{i}$ 's integer.

Example 7: We apply the knapsack approximation to the seven-cell system, shown in Fig. 2, with the traffic pattern $\mathrm{p}=\left(\frac{1}{8}, \frac{1}{8}, \frac{1}{8}, \frac{1}{8}, \frac{1}{8}, \frac{1}{8}, \frac{1}{4}\right)$. For this system $\mathrm{b}=\left(\frac{1}{2}, \frac{1}{2}, \frac{1}{2}\right.$, $\frac{1}{2}, \frac{1}{2}, \frac{1}{2}, 1$ ), for all $r$. We choose $n=10$ and calculate the blocking probability in each cell. These values, along with the exact blocking probabilities are shown in Fig. 7. We see that the approximation works well even for such a small number of channels. The approximation becomes better with increasing $n$, as we noticed in the previous example, where the exact blocking probabilities could be calculated for larger $n$ as well.

Theorem 4: The knapsack approximation in cellular networks is asymptotically correct. 


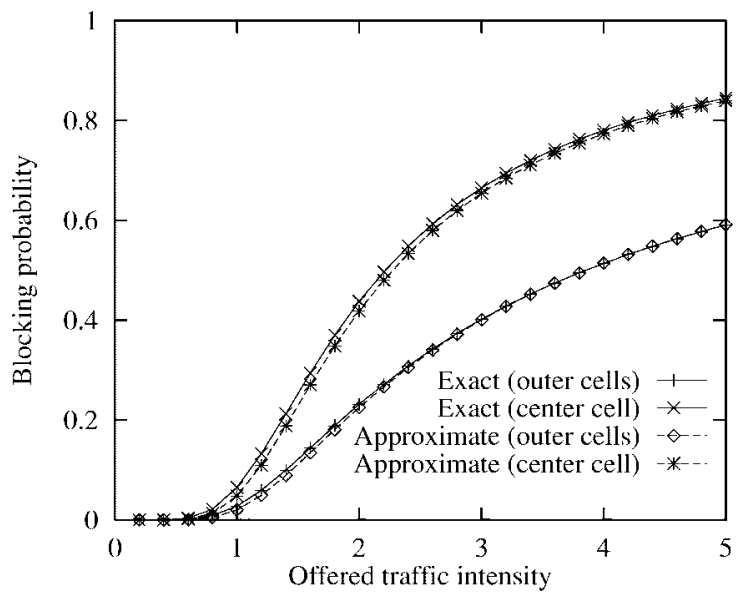

Fig. 7. The knapsack approximation in the seven-cell system for ten channels.

Proof: Kelly [7] considers the following dual optimization problem for communication networks with the links indexed by $j$ and the routes by $i$, where a call on route $i$ requires $a_{i j}$ circuits from link $j$ in the route:

$\min \sum_{i} \nu_{i} \exp \left(-\sum_{j} y_{j} a_{i j}\right)+\sum_{j} y_{j} C_{j}$, subject to $y \geq 0$.

This equation is of the form of the dual problem (25) in the cellular network, with only one link with parameter $y$. All routes consist of just this link and a call on route $i$ requires $b_{i}$ circuits from this link. The $b_{i}$ 's are known. Thus, in the asymptotic case, the blocking probability in cell $i$ is equal to the blocking probability of a call of class- $i$ in this one link communication network.

The knapsack approximation does not give very good results when applied to systems where the set of active MIS's does not remain unique for slight changes in the traffic pattern. We can view this approximation as considering only one active inequality out of several that define the state space in implicit form (see Section II-C). But in the degenerate case, there may be more than one inequality which is active. In this case we can modify the approximation by taking more than one link in series as follows.

\section{B. Multiple-Link Approximation}

We solve the dual problem with many small random perturbations of the traffic pattern $\mathbf{p}$ at the given offered traffic $r$, and thus get a set of link usage vectors $\mathbf{b}^{(1)}, \mathbf{b}^{(2)} \ldots$, etc. This gives a system of linear inequalities of the form $\sum_{i} b_{i}^{(k)} z_{i} \leq n$. We can now eliminate implicit inequalities from this system using the well known Fourier elimination technique (see [12]). From the rest of the inequalities we form a communication network with $K$ links in series, such that a connection of class- $i$ requires $b_{i}^{(k)}$ circuits from link $k, k=1, \cdots, K$. Using the Erlang fixed point approximation [7] or the Knapsack approximation [11] we can calculate the blocking probability of a class- $i$ connection and approximate it to the blocking probability in cell $i$.

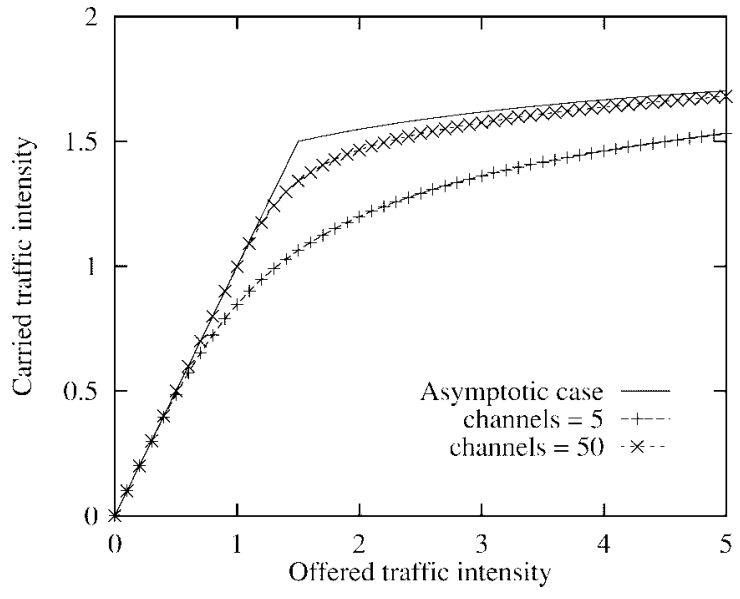

Fig. 8. Performance of MPA in linear three-cell system.

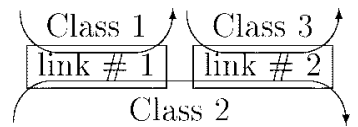

Fig. 9. The multiple-link approximation to the three-cell system.

For $r \leq r_{0}$, we form this communication system with many small random perturbations of the traffic pattern at $r_{0}(\mathbf{p})+\epsilon$, and use the following linear programming approximation to the dual problem:

$$
\max \sum_{i=1}^{N} r_{i} b_{i}
$$

subject to

$$
\sum_{i=1}^{N} a_{i j} b_{i} \leq 1, \quad j=1, \cdots, M
$$

and

$$
b_{i} \geq 0, \quad i=1, \cdots, N \text {. }
$$

Example 8: Consider the linear three-cell system, shown in Fig. 1, with uniform traffic. MIS for this system are $\{2\}$ and $\{1,3\}$. The dual optimization problem for this system gives $\mathrm{b}=\left(\frac{1}{2}, 1, \frac{1}{2}\right)$. With small random perturbations in traffic pattern, the solution of (36) gives

$$
\mathbf{b}=\left\{\begin{array}{l}
\left(\frac{1}{2}, 1, \frac{1}{2}\right) \\
(1,1,0) \\
(0,1,1) .
\end{array}\right.
$$

We see that the inequality given by the first vector is implicit. Hence, the equivalent communication system is as shown in Fig. 9.

In this case, the approximation is exact, as these inequalities completely specify the state space for this system. Performance of MPA in the linear three-cell system is shown in Fig. 8.

Example 9: Consider the 16-cell system, shown in Fig. 4, with uniform traffic. In Fig. 10, we plot the values of $b_{i}$ 's at the solution of the dual optimization problem (25) for various values of the offered traffic $r$. The LP (36), solved at $r_{0}(\mathrm{p})+\epsilon$, with small random perturbations of the traffic pattern, does not 


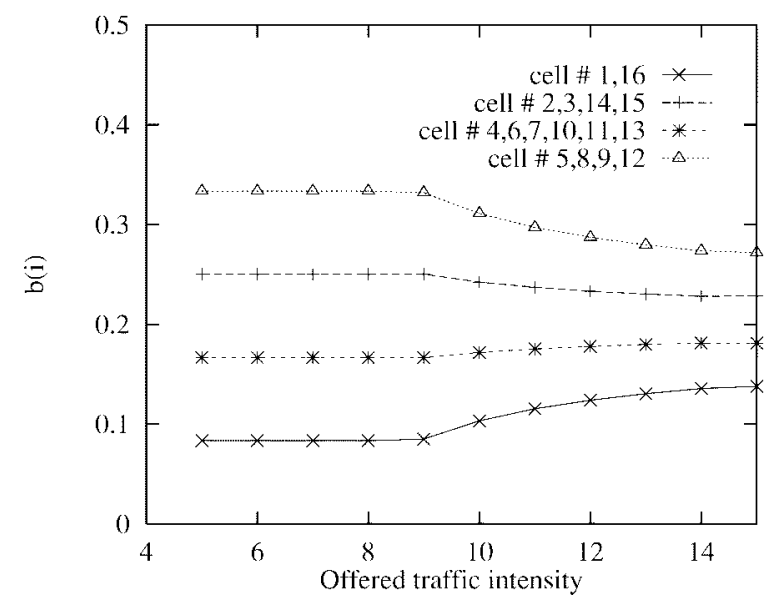

Fig. 10. Change in $b_{i}$ 's with $r$ in the 16-cell system.

change $\mathrm{b}$, which is

$$
b_{i}= \begin{cases}\frac{1}{12}, & i=1,16 \\ \frac{2}{12}, & i=4,6,7,10,11,13 \\ \frac{3}{12}, & i=2,3,14,15 \\ \frac{4}{12}, & i=5,8,9,12 .\end{cases}
$$

Thus, the single-link knapsack approximation should give a good estimate of the blocking probabilities in this system, for offered traffic $r<8$. In Fig. 12, we use the knapsack approximation to calculate the performance of MPA, in this system, for $n=20$ and 100 .

\section{COMParison With Other Algorithms}

In this section, we compare the performance of MPA with two other specific channel assignment algorithms.

1) Asymptotically Optimal FCAA: Derived in [1], in which a fixed channel assignment to the cells is found by solving a linear program. Asymptotically, this assignment achieves the highest possible carried traffic intensity [1]. The algorithm can be extended to the finite channel case by giving the cells the same fraction of total available channels. The carried traffic in cell $i$, when a fraction $x_{i}$ of the channels is assigned to it, is given by

$$
C\left(p_{i} r n, x_{i} n\right)=p_{i} r n\left[1-P_{b}\left(p_{i} r n, x_{i} n\right)\right]
$$

where $P_{b}(\rho, n)$ is given by the Erlang $B$ formula

$$
P_{b}(\rho, n)=\frac{\rho^{n} / n !}{\sum_{k=1}^{n} \rho^{k} / k !} .
$$

From this we calculate the total carried traffic in this case.

2) Greedy DCAA: Assigns the first available channel (channels are numbered from one through $n$ ) in the cell to an arriving call, without violating any of the channel reuse constraints. We have simulated the performance of

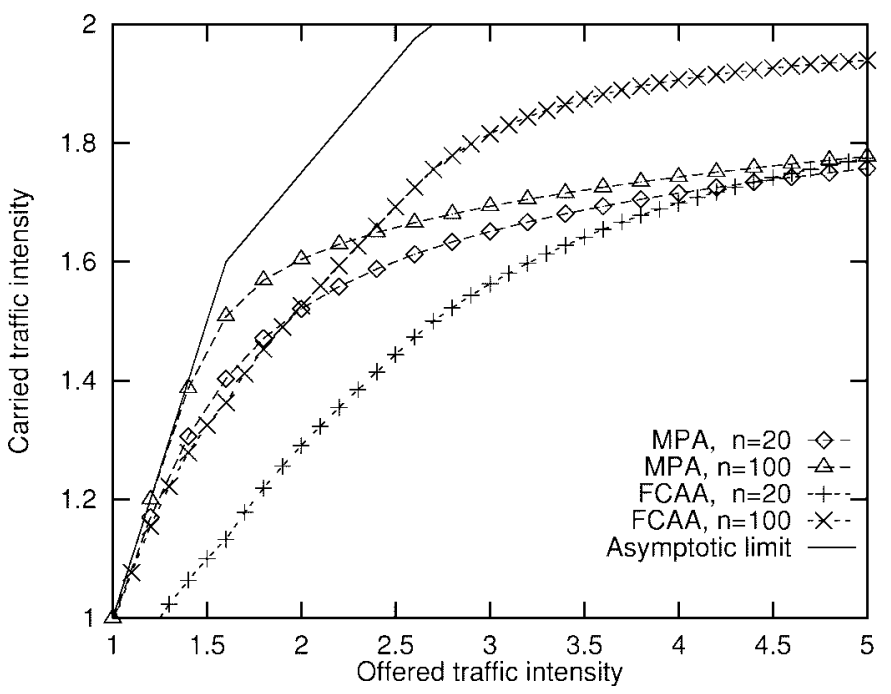

Fig. 11. Performance of MPA and FCAA in the seven-cell system.

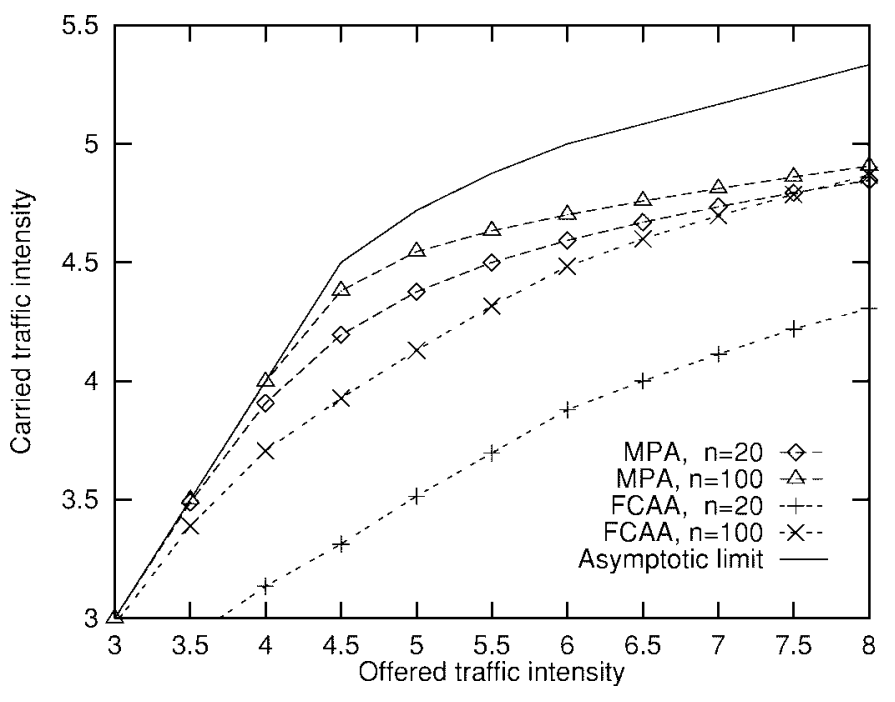

Fig. 12. Performance of MPA and FCAA in the 16-cell system.

this algorithm, assuming call durations are exponentially distributed.

We present the numerical results in Figs. 11-14. We compute the exact performance of MPA in the linear three-cell but use the knapsack approximation in the seven-cell and the 16-cell systems, since the large state space prohibits exact calculation.

- Fig. 11 compares the performance of the MPA and the FCAA in the seven-cell system with the traffic pattern as in the example for $n=20$ and 100 .

- Fig. 12 compares the performance of the MPA and the FCAA in the 16-cell system with uniform traffic, for $n=$ 20 and 100.

- Fig. 13 compares the performance of the MPA and the DCAA in the linear three-cell system with uniform traffic, for $n=20$ and 100 .

- Fig. 14 compares the performance of the MPA and the DCAA in the seven-cell system with the traffic pattern as in the example on for $n=20$ and 100 . 


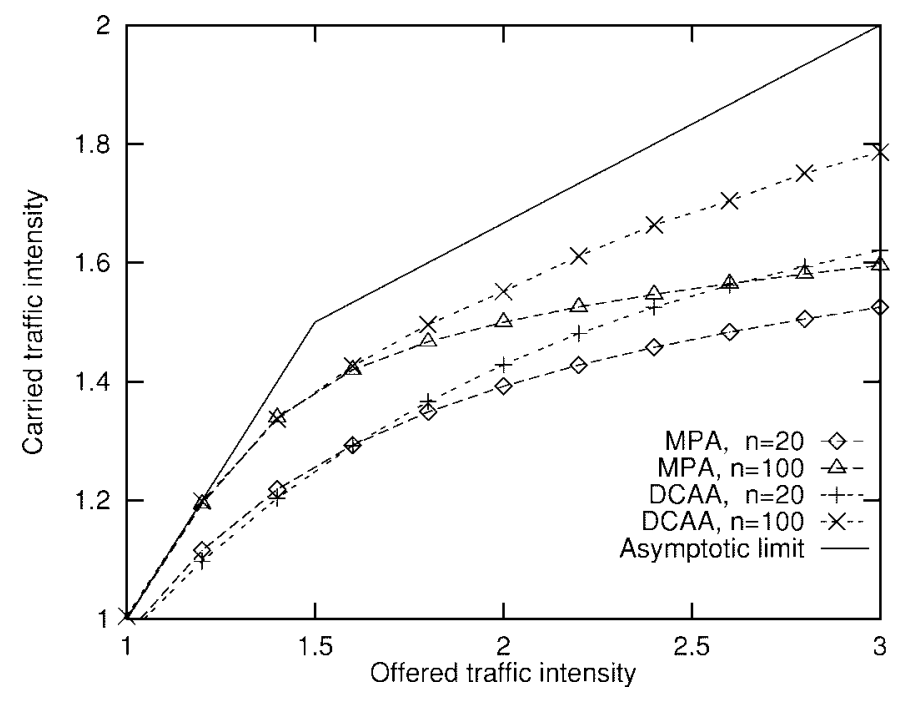

Fig. 13. Performance of MPA and DCAA in the linear three-cell system.

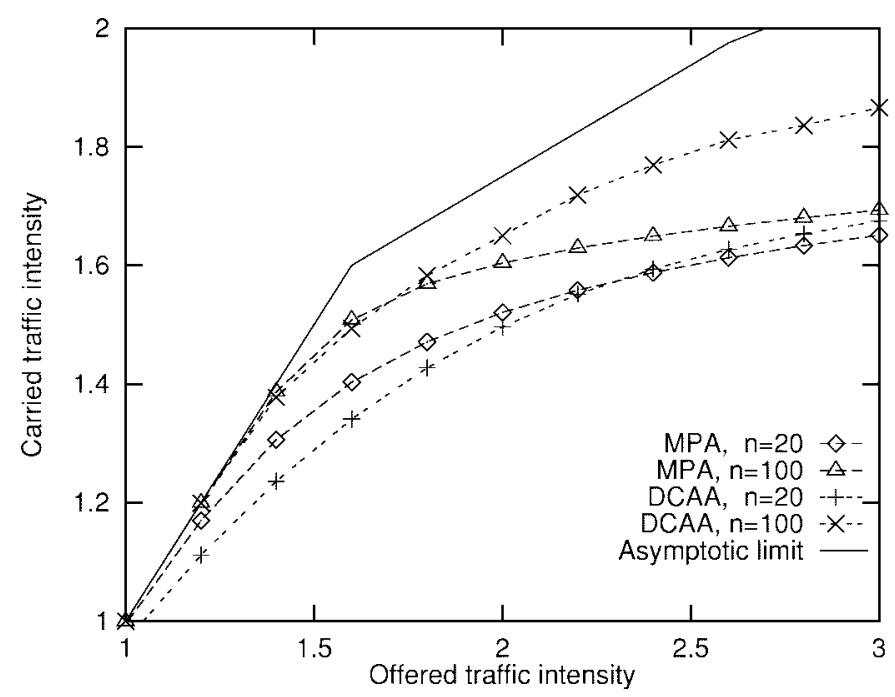

Fig. 14. Performance of MPA and DCAA in the seven-cell system.

From these figures, we observe the following.

1) For light traffic, MPA performs much better than FCAA and better than DCAA. But as the traffic gets heavier, the performance of these algorithms overtakes that of MPA.

2) The improvement due to MPA is more when the number of available channels is less.

3) For each of FCAA and DCAA, there exists a unique crossover offered traffic $r>r_{0}$ at which the performance of MPA becomes worse. This crossover point is closer to $r_{0}$, the greater the number of channels. In the limit it converges to $r_{0}$.

Motivated by these numerical results, we propose the following conjecture.

Conjecture 1: In a cellular system, with offered traffic $r \leq r_{0}$, for any $n$, MPA is the optimal channel assignment algorithm, in terms of the total traffic carried by the system.

We expect the cellular system to be designed such that the offered traffic is less than the capacity ${ }^{6}$ of the system. If this is the case, our conjecture implies that for any finite number of channels, MPA would be the best channel assignment algorithm.

It is worth mentioning that algorithms which give close to optimal performance for high traffic are unfair to certain cells in the system. For example, the optimal FCAA blocks all calls in cell 2 , in the three-cell system, for $r>3$. Thus, when the traffic pattern is unknown, we can say that MPA is fair, in the sense that it gives equal importance to calls in all cells, and it never blocks the calls in one cell completely.

Though we have not considered handoffs in our analysis, we expect MPA to perform better than other algorithms in terms of handoff call dropping probability at least in light to moderate traffic. This is because MPA can always honor a handoff request, in any state, if any other algorithm can do so. Moreover, repacking will provide more room for the handoff request, in light to moderate traffic.

\section{IMPLEMENTATION OF MPA}

We have seen that MPA is better than other algorithms in terms of the total traffic carried for a finite number of available channels, at least for modest offered traffic. This is the region of most interest to us, as blocking probabilities in this region remain within acceptable limits. But its computational complexity makes it almost impractical to implement MPA in large 2-D cellular systems.

In the previous sections, our work relied only on the mathematical property of MPA, viz., this accepts a call if and only if the resulting state is in the state space. Questions such as the channel to be assigned to the new call, other calls which should be reassigned channels, and the actual reassignment remain unanswered. In this section, we devise a strategy to implement MPA in an arbitrary cellular system.

\section{A. MPA Strategy}

We base the MPA implementation on virtual channel assignment to MIS's. The total number of calls, which are reassigned a channel, is an important factor while implementing MPA, because each such reassignment must be accompanied by some overhead information and system time. More the number of reassignments, the less efficient the system becomes, and so we must try to minimize them.

Let us assume that the arrival of the $k$ th customer occurs in cell $i$. We denote the state of the system just before the arrival of this customer by $\mathbf{z}^{-}(k)$ and the virtual assignment vector by $\mathbf{Z}(k)$. We define a new vector $\mathbf{z}^{+}(k)$, such that

$$
z_{l}^{+}(k)= \begin{cases}z_{l}^{-}(k)+1, & \text { if } l=i \\ z_{l}^{-}(k), & \text { otherwise. }\end{cases}
$$

If the call is blocked, the assignment vector $\mathbf{Z}(k)$ is not changed. But if the call is accepted, this might have changed. When a departure occurs, we do not repack calls, and $\mathbf{Z}(k)$ remains the same after a departure.

\footnotetext{
${ }^{6}$ Capacity denotes the maximum offered traffic up to which the optimal channel assignment algorithm blocks no calls asymptotically; see [1] for more details.
} 
The channels are numbered from one to $n$. Channel $c$, $c=1, \cdots, n$, is virtually assigned to an MIS $M_{c}, M_{c}=$ $1, \cdots, M$. We describe the actual assignment by a binary $N \times n$ matrix $\left(m_{i c}\right)$, such that

$$
m_{i c}= \begin{cases}1, & \text { if channel } c \text { is assigned to a call in cell } i \\ 0, & \text { otherwise. }\end{cases}
$$

We denote the set of cells using channel $c$ by $\mathbf{m}_{c}$. It is easy to see that $m_{i c}=0$, if $a_{i M_{c}}=0$.

Step 1) When a call arrives in the system, we check whether assignment $\mathbf{Z}^{(k)}$ satisfies $\mathbf{z}^{+}(k)$. If it does, we need not repack. The call is assigned the first channel (which must exist) satisfying

$$
a_{i M_{c}}=1 \text { and } m_{i c}=0 .
$$

Step 2) Even when $\mathbf{Z}^{(k)}$ does not satisfy $\mathbf{z}^{+}(k)$, we may be able to assign a channel to the new call without repacking. Let $V_{j}$ denotes the $j$ th maximal independent set. If there exists a channel $c$, such that

$$
\begin{aligned}
& \mathbf{m}_{c} \subset V_{M_{c}}, a_{i M_{c}}=0 \\
& \mathbf{m}_{c} \subset V_{j}\left(j \neq M_{c}\right), a_{i j}=1
\end{aligned}
$$

then the channel $c$ is virtually reassigned to the MIS $j$, and the call is assigned the channel $c$ without repacking.

Step 3) If no channel satisfies Steps 1) and 2), the call can be honored with repacking, only if there exists another assignment vector $\mathbf{Z}^{(k+1)}$, such that

$$
\begin{aligned}
& \sum_{j=1}^{M} Z_{j}^{(k+1)} \leq n \\
& \sum_{j=1}^{M} a_{l j} Z_{j}^{(k+1)} \geq z_{l}^{+}(k), \quad l=1, \cdots, M \\
& \Rightarrow \sum_{j=1}^{M} a_{l j}\left(Z_{j}^{(k+1)}-Z_{j}^{(k)}\right) \\
& \geq z_{l}^{+}(k)-\sum_{j=1}^{M} a_{l j} Z_{j}^{(k)}, \quad l=1, \cdots, M .
\end{aligned}
$$

We rewrite the last inequality in terms of difference variables $d_{j}\left(=d_{j}^{+}-d_{j}^{-}, d_{j}^{+} \in \mathcal{Z}_{+}, d_{j}^{-} \in \mathcal{Z}_{+}, d_{j}^{+} d_{j}^{-}=0\right)=$ $Z_{j}^{(k+1)}-Z_{j}^{(k)}$ and replace the right-hand side by $u_{l}$, for simplicity. We note that

$$
\begin{aligned}
u_{l} & =1, & & \text { if } l=i \\
& \leq 0, & & \text { otherwise. }
\end{aligned}
$$

$d_{j}$ denotes the number of channels being transferred to the MIS $j$. If $d_{j}^{+}>0$, the MIS $j$ should be reassigned $d_{j}^{+}$more channels, while if $d_{j}^{-}<0, d_{j}^{-}$channels should be deducted from the MIS $j$.

The total number of calls, which are reassigned a channel, is an important factor while implementing MPA, and we must try to minimize them. Actual reassignments are directly correlated with the number of virtual reassignments to MIS's.
To minimize the total number of channels deducted from MIS's, we solve the following integer linear program.

1) Integer Linear Program:

$$
\min \sum_{j=1}^{M} d_{j}^{-}
$$

subject to

$$
\begin{gathered}
\sum_{j=1}^{M} a_{l j}\left(d_{j}^{+}-d_{j}^{-}\right) \geq u_{l} \quad l=1, \cdots, N \\
0 \leq d_{j}^{-} \leq Z_{j}^{(k)}, \quad d_{j}^{+} \geq 0, \quad j=1, \cdots, M \\
\sum_{j} d_{j}^{+} \leq \sum_{j} d_{j}^{-} .
\end{gathered}
$$

At the solution of this ILP, $\mathbf{d}$ must satisfy

$$
d_{j}^{+} d_{j}^{-}=0 \text { and } \sum_{j} d_{j}^{+}=\sum_{j} d_{j}^{-} .
$$

After solving the ILP, we have a set of $\beta=\sum_{j} d_{j}^{-}$ candidate MIS's from which a channel must be deducted, and must be given to a set of $\beta$ other MIS's (counting each MIS the number of times a channel must be reassigned to it). We could randomly choose disjoint pairs from these two sets and transfer channels in those pairs, though we can further minimize repacking by forming these pairs intelligently, as we will now describe.

If a channel is to be reassigned to the $j$ th MIS from the $i$ th MIS, then calls which are using this channel and are not in the new MIS $j$, must be reassigned a channel. In the worst case, this number could be as large as $\left|V_{j} \backslash V_{i}\right|,{ }^{7}$ where $V_{j}$ denotes the $j$ th maximal independent set. Thus, the worst case repacking for each pair of MIS is denoted by

$$
w_{i j}=\left|V_{j} \backslash V_{i}\right|, \quad i, j=1, \cdots, M
$$

With this development, we can formulate our problem of repacking minimization as a weighted matching problem in a complete bipartite graph with $2 \beta$ vertices.

We have two sets of vertices $V$, corresponding to the MIS's from which channel should be deducted, each MIS is counted $d_{j}^{-}$number of times, and $U$, corresponding to the MIS's to which channel is to be assigned, each MIS counted $d_{j}^{+}$ number of times, of cardinality $\beta$. From each vertex $v_{i} \in V$, $i=1, \cdots, \beta$, there is an edge with the weight $w_{i j}$ to all vertices $u_{j} \in U, j=1, \cdots, \beta$. Now we find $\beta$ ordered pairs $\left(v_{i}, u_{j}\right)$, such that no two pairs share the same vertex and the total weight associated with the set of pairs is minimized.

The weighted matching problem in a complete bipartite graph is a well-addressed problem in the literature of combinatorial optimization and graph theory. We can use the Hungarian method, see [13, pp. 247-271], to solve this problem, which takes $O\left(\beta^{3}\right)$ mathematical operations.

Now we know the pairs of MIS's for virtual transfer of channels. Now we can choose a channel assigned to the donor MIS to minimize actual repacking. Note that for minimum

$$
{ }^{7} A \backslash B=\{v: v \in A, v \notin B\} .
$$




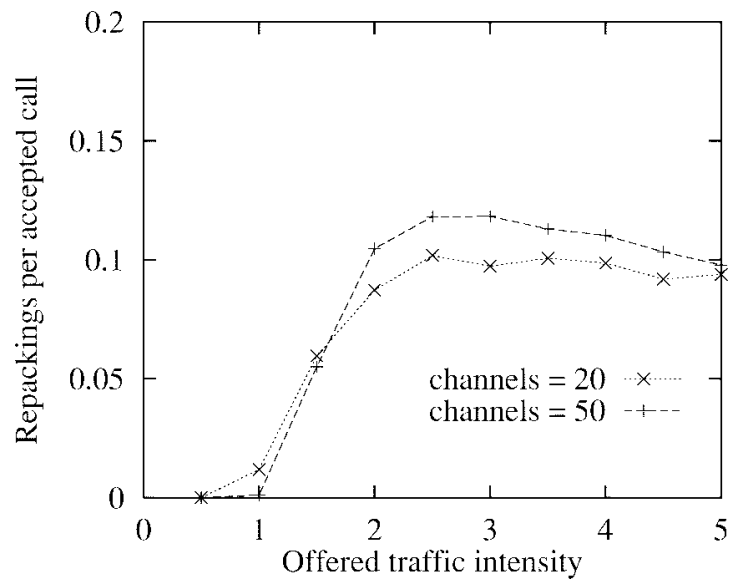

Fig. 15. Number of repackings per accepted call $\Psi(r, n)$ in the linear three-cell system.

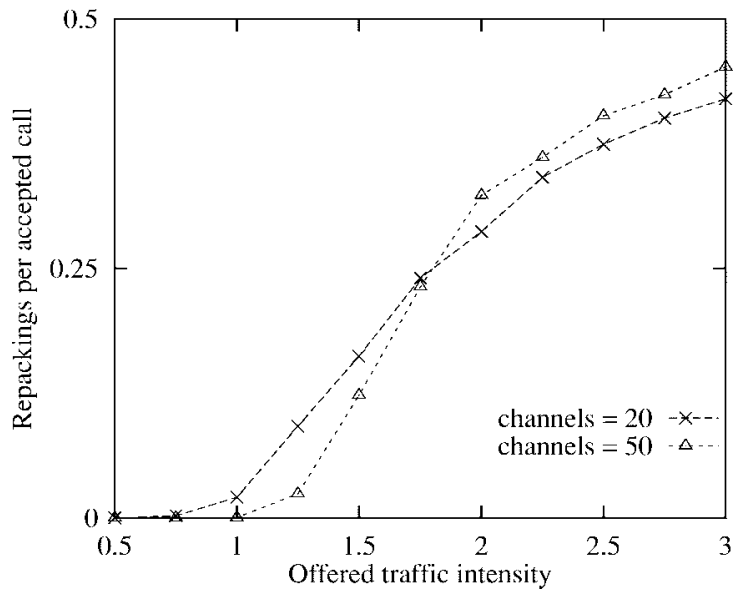

Fig. 16. Number of repackings per accepted call $\Psi(r, n)$ in the seven-cell system.

repacking this strategy is only close to optimal, e.g., we could use the cost matrix derived from the current actual assignment [matrix $\left(m_{i c}\right)$ ] to minimize the actual cost. But considering the latter improves the results by only a small factor, because when repacking is required, most of the channels are already densely packed, and hence the worst case cost estimate is not much different from the actual cost.

\section{B. Numerical Results}

We simulate the performance of the cellular systems using the efficient MPA strategy. In previous sections, we have analyzed the performance mainly in terms of the blocking probability and the total carried traffic. Here, we concentrate on the other important aspect of MPA, viz., the number of calls reassigned a channel to accommodate a new call.

The average number of repackings per accepted call, denoted by $\Psi(r, n)$, is shown in Figs. 15 and 16 for the linear three-cell system and the symmetrical seven-cell system, respectively, for 20 and 50 available channels.

From these figures, we observe the following.

1) $\Psi(r, n)$ Versus $r$ : For very low offered traffic, $\Psi(r, n) \approx 0$, because even DCAA [Steps 1) and 2) of

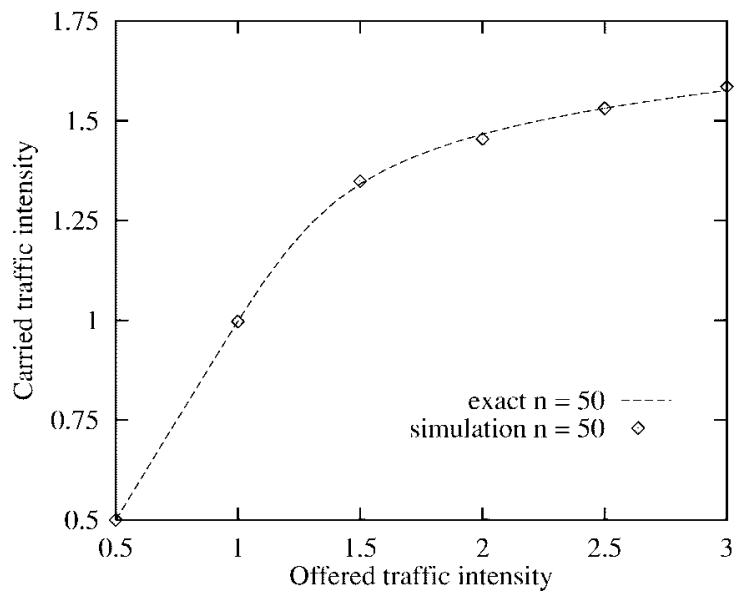

Fig. 17. Comparison of the exact performance and the simulation results in the linear three-cell case, for $n=50$.

MPA] has negligible blocking, so that most of the accepted calls do not perform Step 3). Then $\Psi(r, n)$ increases with increasing offered traffic.

2) $\Psi(r, n)$ Versus $n$ : Average repacking increases with the number of available channels $n$, because more the number of channels, more the scope for the new call to get a channel free after repacking. But for low offered traffic the behavior is the converse. This is because the blocking probability decreases with increasing $n$, and, thus, the probability that the call will get a channel in the first two steps of MPA increases with $n$, for low offered traffic.

3) $\Psi(r, n)$ with System Size: $\Psi(r, n)$ increases with increase in the system size. We expect that this number increases without bound as the system gets larger.

We compare the exact and the simulation results in Figs. 17 and 18 , for the linear three-cell system and the seven-cell system, respectively, with 50 channels. Fig. 19 shows the distribution of number of calls for number of repackings required. The worst case number of repackings in the example systems we consider (obtained by simulation) are listed in Table I.

TABLE I

\begin{tabular}{l|c}
\hline Cellular systems & Worst case repacking \\
\hline $\begin{array}{l}\text { linear } n \text { cell system } \\
\text { symmetrical } 7 \text { cell system }\end{array}$ & $\lfloor(n-1) / 2\rfloor$ \\
\hline
\end{tabular}

\section{Approximate MPA}

Computational complexity is a major concern while implementing an algorithm. Steps 1) and 2) and the weighted matching problem in Step 3) take $O(n), O(M \times n)$, and $O\left(\nu^{3}\right), \nu \ll M$, mathematical operations, respectively. Thus, the integer linear program, with $2 M$ variables and $2 M+N+1$ inequalities, which is known to be NP complete, is the most crucial element of the efficient MPA.

We suggest the following approximations to the efficient MPA strategy to make it computationally a feasible real-time algorithm and end the section.

1) Blocking the Call Beforehand: We derive the system of linear inequalities for the network, of the form (5), derived 


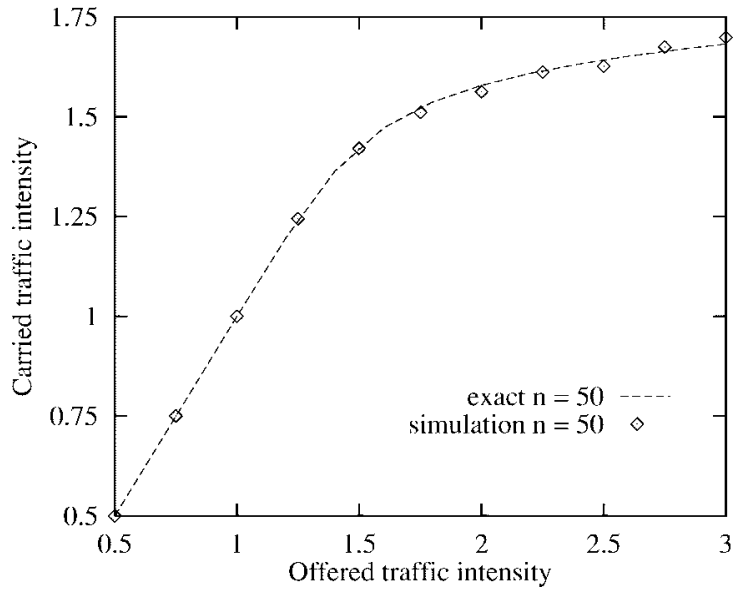

Fig. 18. Comparison of the exact performance and the simulation results in the seven-cell case, for $n=50$.

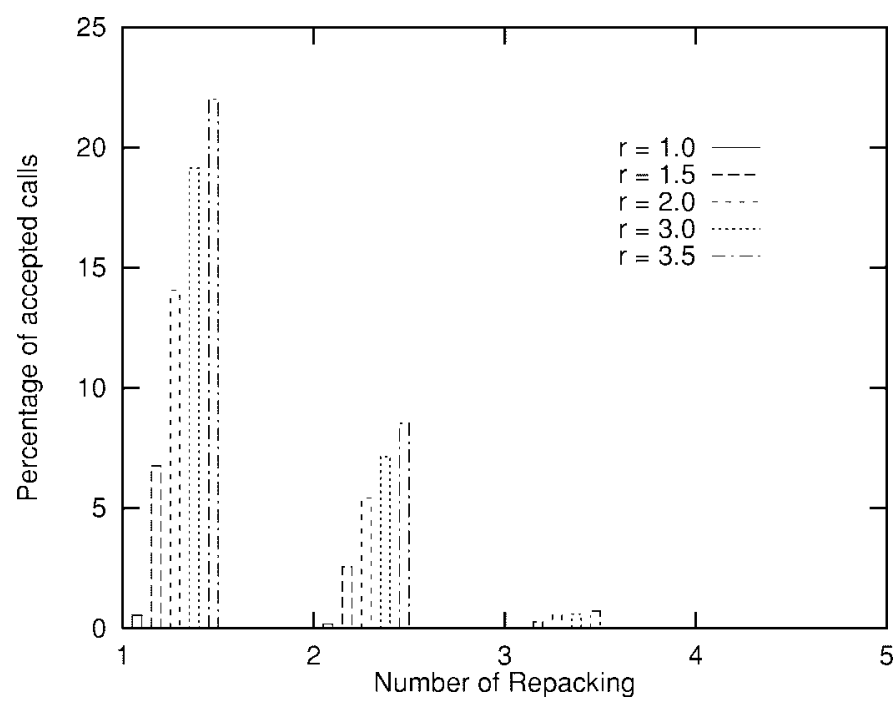

Fig. 19. Histogram depicting percentage of calls which required one, two, three, or four repackings in the seven-cell case, for $n=20$.

in the Section II-C2. We may consider the call only if the resulting state satisfies this system of linear inequalities and otherwise block it without further consideration. This will reduce the computational burden on the system very much because most of the time the call considered for Step 3) of the MPA is blocked.

2) LP instead of ILP: Linear program is known to be computationally very easy compared to the ILP. We may solve the program in Step 3), relaxing the constraints of $d_{j}^{+}$and $d_{j}^{-}$being integer. Then we may round them to get a feasible integer solution.

3) Only Active MIS's: The total number of MIS's $M$ is exponential in the number of cells in the system $N$. We may consider only those MIS's which are given nonzero fraction of channels asymptotically at the solution of primal optimization problem, and neglect other inactive MIS's. Thus, the computational complexity of almost all the steps gets reduced significantly.

\section{CONCLUSION}

We have analyzed the performance of the MPA algorithm for an arbitrary cellular system with a general traffic pattern. In the asymptotic limit, the blocking probabilities can be derived using a nonlinear optimization problem. We notice that up to a point $r_{0}$ (which can be derived using an LP) MPA performs without blocking and tracks the achievable limiting performance of the system (derived in [1]), but after this point performance is poor compared to the limiting performance. The cellular system with a finite number of available channels can be approximated by a simple fixedrouting communication network and the blocking probabilities in the cells can be approximated by blocking probabilities of the classes of connections on the network.

We see that the carried traffic for the MPA converges to its asymptotic limit faster than other algorithms. Numerical results motivate us to conjecture that MPA is an optimal channel assignment algorithm for a finite number of channels for offered traffic less than $r_{0}$. We suggest a procedure to implement MPA, based on the mathematical framework. The average number of repackings per accepted call and the complexity of the implementation increase with system size. But with some approximations, MPA can be a practical algorithm for small to moderate sized systems.

\section{ACKNOWLEDGMENT}

The authors would like to thank the referees for their comments which have improved the presentation of this paper.

\section{REFERENCES}

[1] R. J. McEliece and K. N. Sivarajan, "Performance limits for channelized cellular telephone systems," IEEE Trans. Inform. Theory, vol. 40, pp. 21-34, Jan. 1994.

[2] D. E. Everitt and N. W. Macfadyen, "Analysis of multicellular mobile radio telephone systems with loss," Br. Telecom Tech. J., vol. 1, no. 2, pp. 37-45, 1983.

[3] E. Del Re, R. Fantacci, and G. Giambene, "Handover and dynamic channel allocation techniques in mobile cellular networks," IEEE Trans. Veh. Technol., vol. 44, pp. 229-237, May 1995.

[4] D. Everitt and D. Manfield, "Performance analysis of cellular mobile communication system with dynamic channel assignment," IEEE Trans. Select. Areas Commun., vol. 7, pp. 1172-1180, Oct. 1989.

[5] P. A. Raymond, "Performance analysis of cellular networks," IEEE Trans. Commun., vol. 39, pp. 1787-1793, Dec. 1991.

[6] S. Jordan and A. Khan, "A performance bound on dynamic channel allocation in cellular systems: Equal load," IEEE Trans. Veh. Technol., vol. 43, pp. 333-344, May 1994.

[7] F. P. Kelly, "Blocking probability in large circuit switched networks," Adv. Appl. Prob., vol. 18, pp. 473-505, 1986.

[8] _L "Loss networks," Ann. Appl. Prob., vol. 1, pp. 317-378, 1991.

[9] S. Sarkar and K. N. Sivarajan, "Hypergraph models for cellular mobile communications," IEEE Trans. Veh. Technol., vol. 47, pp. 460-471, May 1998.

[10] J. S. Kaufman, "Blocking in a shared resource environment," IEEE Trans. Commun., vol. COM-29, pp. 1474-1487, Oct. 1981.

[11] S.-P. Chung and K. W. Ross, "Reduced load approximation for multirate loss networks," IEEE Trans. Commun., vol. 41, pp. 1222-1231, Aug. 1993.

[12] V. Chandru, "Variable elimination in linear constraints," Computer J., vol. 36, no. 5, pp. 463-472, 1993.

[13] C. H. Papadimitriou and K. Steiglitz, Combinatorial Optimization: Algorithms and Complexity. Englewood Cliffs, NJ: Prentice-Hall, 1982. 


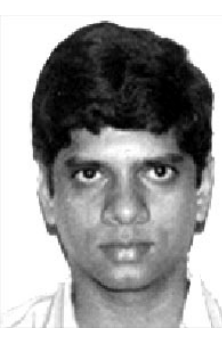

Ashutosh Kulshreshtha was born in Agra, India, on October 16, 1974. He received the B.E. degree in electronics and communications from the Maulana Azad College of Technology (REC), Bhopal, India, in 1995 and the M.E. degree in electrical communication engineering from the Indian Institute of Science, Bangalore, India, in 1997.

From February 1997 to May 1998, he was with Hughes Software System, Gurgaon, India. Since May 1998, he has been with the IBM Solutions Research Center, New Delhi, India, as a Technical Staff Member. His research interests are in the area of cellular and computer networks.

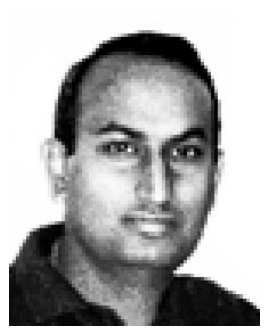

Kumar N. Sivarajan (S'88-M'90) was born on October 23, 1965, in Bangalore, India. $\mathrm{He}$ received the $\mathrm{B}$.Tech. degree in electrical engineering (electronics) from the Indian Institute of Technology, Madras, India, in 1987 and the M.S. and Ph.D. degrees in electrical engineering from the California Institute of Technology, Pasadena, in 1988 and 1990, respectively.

From 1990 to 1994 , he was with the IBM

Thomas J. Watson Research Center, Yorktown Heights, NY. Since October 1994, he has been with the Electrical Communication Engineering Department, Indian Institute of Science, Bangalore, where he is presently an Associate Professor. He was an Academic Visitor with the IBM T. J. Watson Research Center during the summers of 1996 and 1997. His research interests include modeling, design, and performance analysis of cellular, optical, and IP/ATM networks.

Dr. Sivarajan is an Editor of the IEEE/ACM TRANSACTIONS ON NETWORKING and an Associate of the Indian Academy of Sciences. He was the recipient of the IEEE Charles LeGeyt Fortescue Fellowship for the academic year 1987-1988, and the corecipient with Dr. Rajiv Ramaswami of the IEEE Communications Society 1996 William R. Bennett Prize Paper Award and the 1997 IEEE W. R. G. Baker Prize Paper Award. He received a Young Engineer Award from the Indian National Academy of Engineering in 1998. 